\title{
Does Antimatter Fall Like Matter?: Simulation of the GBAR Experiment
}

\author{
Olivier Rousselle, Pierre Cladé, Saïda Guellati, Romain Guérout, and Serge Reynaud \\ Laboratoire Kastler Brossel, Sorbonne Université, ENS-PSL, Collège de France, CNRS, 75005 Paris, France
}

\begin{abstract}
One of the main questions of fundamental physics is the action of gravity on antimatter. We present here the simulation of the last part of the experiment GBAR at CERN, i.e. the measurement of the free fall acceleration $\bar{g}$ of antihydrogen atoms in the gravitational field of the Earth. It includes the Monte-Carlo generation of trajectories and the analysis leading to the estimation of $\bar{g}$. A precision of the measurement beyond the \% level is confirmed when taking into account the experimental design.
\end{abstract}

Keywords: antimatter, gravity, statistics, interferences

DOI: $10.31526 /$ ACP.BSM-2021.35

\section{INTRODUCTION}

The asymmetry between matter and antimatter observed in the Universe is one of the fundamental problems of modern physics challenging the Standard Model. Hints on this puzzle are looked for in experiments testing CPT symmetry between matter and antimatter as well as the interaction of antimatter with gravity [1,2]. CPT tests are already quite precise but tests on antimatter gravity have currently a limited precision, with the sign of gravity acceleration not yet known experimentally [3].

The Einstein Equivalence Principle, a cornerstone of General Relativity, implies that all objects fall with the same acceleration in a given gravity field. This Weak Equivalence Principle has been tested with high precision [4], and ambitious projects are developed at CERN facilities to produce low energy antihydrogen with the aim of measuring the free fall of antihydrogen atoms. Among them, the GBAR experiment (Gravitational Behaviour of Antihydrogen at Rest) aims at measuring $\bar{g}$, the gravity acceleration of antihydrogen in the Earth gravity field, by timing the free fall of ultra-cold $\bar{H}$ atoms [5].

\section{PRESENTATION AND MODELLING OF GBAR EXPERIMENT}

The principle of GBAR experiment is based upon the original idea of Hänsch and Walz [6]. Antihydrogen ions $\bar{H}^{+}$are cooled in an ion trap by using laser cooling techniques. The excess positron of $\bar{H}^{+}$is photodetached with a laser, forming a neutral antihydrogen atom $\bar{H}$, and this marks the start of the free fall time [7]. At the end of this free fall, the antiatom annihilates on the detection surface of the free fall chamber producing secondary particles, the detection of which makes it possible to reconstruct the time $T$ and position $R$ of the annihilation event (see fig.1). The free fall acceleration $\bar{g}$ is deduced from a statistical analysis of annihilated events.

The dimensions of the cylindrical chamber are the free fall height $H_{f}=30 \mathrm{~cm}$, the ceiling height $H_{c}=30 \mathrm{~cm}$, and the chamber radius $R_{c}=25 \mathrm{~cm}$. It is expected that about $N=1000$ antiatoms will be used in the experiment. In the simulations, we assume that free fall acceleration of antihydrogen has its standard value and study the precision for its measurement.

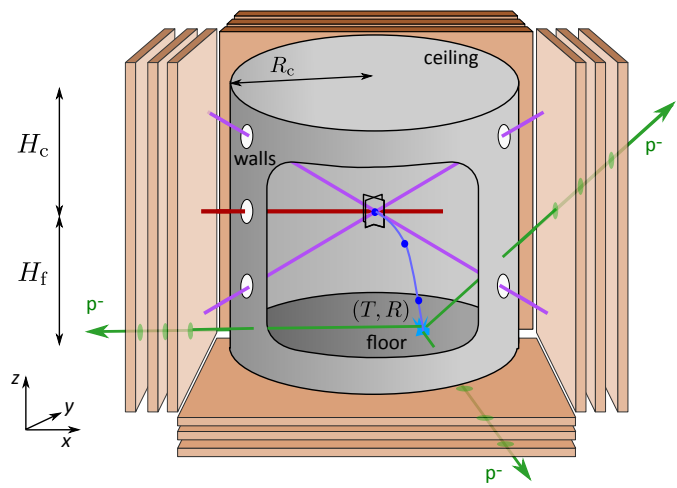

FIGURE 1: Principle of the free fall measurement in the GBAR experiment. Details of the figure: cylindrical chamber (in grey); an example of $\bar{H}$ trajectory from trap to detection surface (in blue); secondary particles produced by the annihilation (in green); Micromegas detectors (in orange). 
The initial wave packet is in the ground state of an harmonic ion trap. It has minimal dispersion identical along the three space directions:

$$
\zeta=\sqrt{\frac{\hbar}{2 m f}}, \Delta v=\frac{\hbar}{2 m \zeta}=\sqrt{\frac{h f}{2 m}}
$$

with $\zeta$ position dispersion, $\Delta v$ velocity dispersion, $f$ trap frequency, $m$ mass of antihydrogen atom and $\hbar$ reduced Planck constant.

After photodetachement, atoms are freely falling in the Earth gravityl field. Each detection point is characterized by its position $(X, Y, Z)$ and time of flight $T$. There is a one to one matching between these parameters and the components of initial velocity, described by classical mechanics. The observable is the particle current $J$ which counts the number of particle per unit of time and per unit of surface (flux):

$$
J(\vec{R}, T)=\frac{m^{3}\left|V_{\perp}\right|}{T^{3}} \Pi_{0}\left(\vec{p}_{0}\right),
$$

with $\left|V_{\perp}\right|$ the speed orthogonal to the surface of detection and $\Pi_{0}$ the initial momentum distribution which results from convolution of initial velocity distribution inside trap and velocity kick induced by photodetachement.

\section{SIMULATIONS AND ESTIMATION OF THE UNCERTAINTY}

In this section, we present two manners of analysing the accuracy to be expected in the experiment. The first one, close to the data analysis to be designed for the experiment, is a numerical Monte-Carlo method. Another one is the analytical Cramer-Rao method. We compare the results of the two methods, and use their good agreement as a cross validation of the results.

\subsection{Monte-Carlo simulation}

Using the standard valuer $g_{0}=9.81 \mathrm{~m} / \mathrm{s}^{2}$, we generate $N=1000$ atoms with random initial velocity $\vec{v}_{0}$. Each atom undergoes a classical free fall, and each detection event is characterized by its impact position $(X, Y, Z)$, its time of flight $(T)$ and the surface it reaches (floor/walls/ceiling). For a random draw of $N$ annihilation events, we calculate the likelihood function:

$$
\mathcal{L}(g)=\prod_{i=1}^{N} J_{g}\left(X_{i}, Y_{i}, Z_{i}, T_{i}\right)
$$

We define the estimator as the mean of the likelihood: $\widehat{g}=\frac{\int g \mathcal{L}(g) d g}{\int \mathcal{L}(g) d g}$.

We repeat this process (generation of events and mean likelihood estimation) a large number $M$ of times, each simulation providing an estimator $\widehat{g}$. An example of random draws is illustrated on fig.2. From the histogram, we extract the mean value (close to $g_{0}$ ) and the standard deviation denoted $\sigma_{g}^{\mathrm{MC}}$.
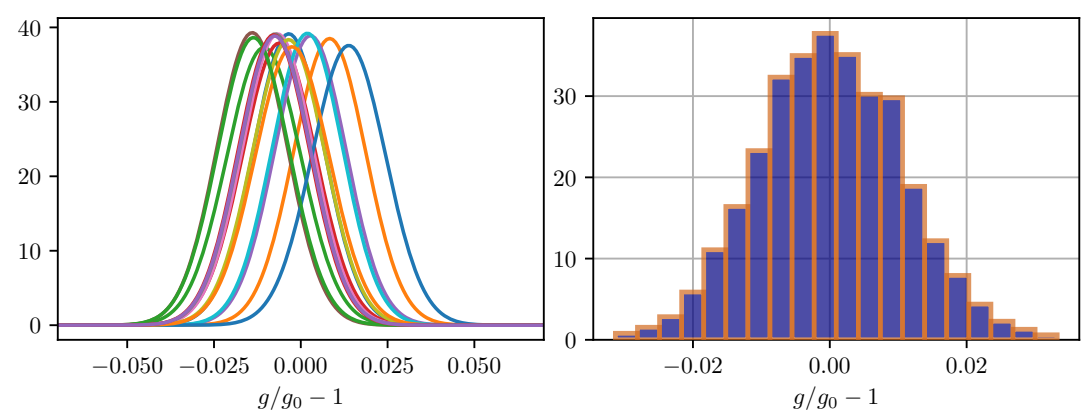

FIGURE 2: Normalized likelihood functions obtained for 15 independent random draws of 1000 atoms (left) and histogram of the estimators $\widehat{g}$ for 5000 random draws (right)

\subsection{Cramer-Rao method}

The Cramer-Rao lower bound for the dispersion $\sigma_{g}^{\mathrm{CR}}$ is deduced from the Fisher information $\mathcal{I}_{g}$ calculated from the $g$-dependence of the distribution:

$$
\mathcal{I}_{g}=\int \mathrm{d} S \mathrm{~d} T \frac{\left(\partial_{g} J_{g}\right)^{2}}{J_{g}}, \quad \sigma_{g}^{\mathrm{CR}}=\frac{1}{\sqrt{\mathrm{NI}_{g}}} .
$$

With the parameters of the design, we get a relative uncertainty of $\sigma_{g} / g$ below $1 \%$, thus confirming the precision goal of the GBAR experiment. We also get $\sigma_{g}^{\mathrm{MC}} \approx \sigma_{g}^{\mathrm{CR}}$, which shows the good statistical efficiency of the analysis method [8]. 


\section{MEASUREMENT WITH QUANTUM INTERFERENCES}

We also propose to improve the accuracy of the measurement by using the idea of quantum techniques drawn from experiments performed on ultracold neutrons [9]. To the previous design, we add a circular reflecting mirror of diameter $d=5 \mathrm{~cm}$ at a distance $h=10 \mu \mathrm{m}$ below the trap (see fig.3). Anti-atoms bounce several times above the mirror thanks to quantum reflection, and the quantum paths corresponding to different GQS (Gravitational Quantum States) interfere [10]. After a free fall of height $H$, we show that the quantum interference pattern on the detector reveals much more information on the value of $\bar{g}$ than the classical one.

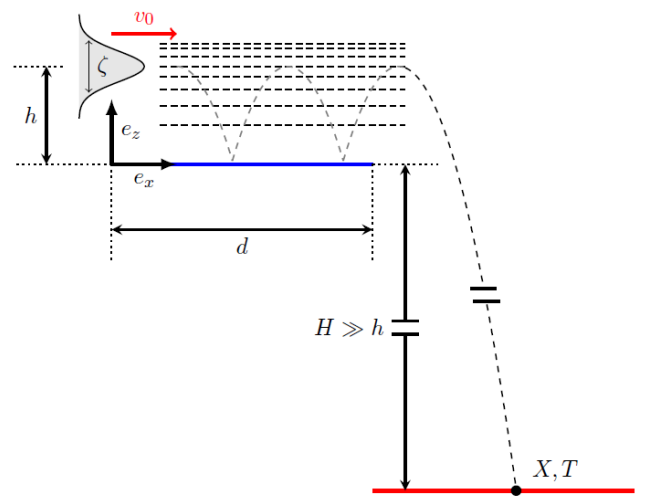

FIGURE 3: 2D schematic representation of the experimental design [10].

The total time $T$ is now the sum of the time above the mirror $t$ and the free fall time $\tau$ and the expression of the annihilation current at the detection is:

$$
J(\vec{R}, T)=\frac{m^{3}\left|V_{\perp}\right|}{\tau^{3}} \Pi_{d}(\vec{p})
$$

where $\Pi_{d}$ is the momentum distribution at the end of the mirror (of radius $d$ ).

We represent in fig.4 the current $J$ obtained, which clearly reveals interference fringes.

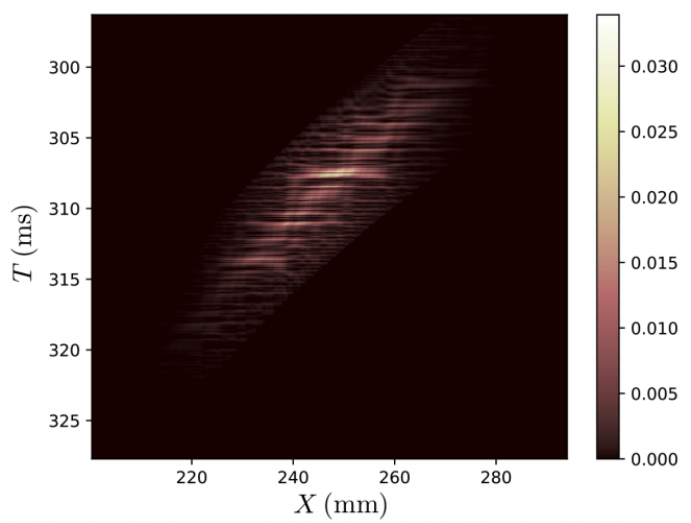

FIGURE 4: Probability current density $J(X, T)$ on the detection plate - with $Y=0$ and $Z=-H[10]$.

Determining the uncertainty of the quantum experiment with the methods described in part 3 , we get a relative uncertainty of $\sigma_{g} / g$ improving the accuracy by a factor of about 1000 compared to the classical timing method.

\section{CONCLUSION}

We have modelled the GBAR free fall chamber by taking into account the main parameters of the experiment. This work aims at preparing the data analysis and finding the optimal parameters for the GBAR experiment.

The Monte-Carlo simulation for $N=1000$ antihydrogen atoms used in the experiment leads to a relative uncertainty of $\sigma_{g} / g$ below $1 \%$, and this result is confirmed by using the analytical Cramer-Rao method.

We also proposed a new method using quantum reflection of $\bar{H}$ atoms above a reflecting mirror followed by a classical free fall. The quantum interference pattern thus obtained brings more information on the value of $\bar{g}$ than the classical method, and then improves the accuracy of the experiment by approximately 3 orders of magnitude. 


\section{ACKNOWLEDGMENTS}

The authors acknowledge insightful discussions with members of the GBAR collaboration.

\section{References}

[1] W.A. Bertsche et al, Journal of Physics B 48, 232001 (2015).

[2] Y. Yamazaki, Proceedings of the Japan Academy B 96, 471-501 (2020).

[3] The ALPHA collaboration and A.E. Charman, Nature Communications 4, 1785 (2013).

[4] P. Touboul et al., Physical Review Letters 119, 231101 (2017).

[5] P. Indelicato et al., Hyperfine Interactions 228, 141-150 (2014).

[6] J. Walz and T.W. H?nsch, General Relativity and Gravitation 36, 561-570 (2004).

[7] P. Pérez et al., Hyperfine Interactions 233, 21-27 (2014).

[8] H. Cramer, Princeton University Press (1946).

[9] V.V. Nesvizhevsky et al., Nature 415, 297-299 (2002).

[10] P.P. Crépin et al., Physical Review A 99, 042119 (2019). 